\title{
Autodependencia tecnológica y cooperación entre los países del Tercer Mundo ${ }^{*}$.
}

Este ensayo trata acerca del rol de la cooperación internacional entre los países del Tercer Mundo con el propósito de alcanzar un desarrollo autodependiente. Se concentra en el rol de la ciencia y la tecnología y examina el contenido del concepto de autodependencia en relación con la tecnología, el cambiante molde de las relaciones internacionales, y las estrategias posibles para la cooperación entre los países subdesarrollados. El trabajo termina con una propuesta para la acción a un nivel institucional.

\section{AUTODEPENDENCIA, DESARROLLO Y TECNOLOGÍA}

Durante los últimos diez años el concepto de autodependencia ha adquirido cada vez más importancia en la conceptualización de los procesos de desarrollo. Ha habido muchas proposiciones sugiriendo cómo este concepto puede ser incorporado en las estrategias de desarrollo, si bien no existe un acuerdo claro acerca de su contenido preciso. Las raíces intelectuales y politicas de la idea de autodependencia abarcan más de un siglo y han emergido en diversas situaciones (siendo testigo de pensamientos utópicos sobre comunidades autónomas), lo que dificulta la identificación de un significado para el concepto de autodependencia que responda a una teoría de desarrollo y se aplique en forma general.

En una revisión de la concepción actual de la autodependencia, Cardettini ${ }^{1}$ rastrea sus orígenes en el pensamiento de Mao y en la filosofía de Ghandi, destacando que ésta se ha expandido -ya sea como una aseveración político-filosófica o como un componente de

\footnotetext{
* Coordinador en el terreno, Proyecto STPI y vicedirector del Conscjo, Instituto Industrial de Tecnología (ITINTEC), Lima, Perú.

* Basado en un trabajo presentado en el $24^{\circ}$ simposio de Pugwash sobre "Auto. dependencia y estratcgia de desarrollo alternativo", Dar es Salaam, junio 1975.

${ }^{1} \mathrm{O}$. Cardettini, Technological Dependence/self reliance: an introductory statement, Proyecto STIPI, Oficina del Coordinador en el terreno, Lima, Perú, abril 1975 .
} 
las estrategias de desarrollo- a países tan diversos como Algeria, Perú, India, Cuba, Tanzania, China y la Costa de Marfil. El origen de esta preocupación puede haber sido el descubrimiento de que la ayuda para el desarrollo era totalmente insuficiente, el deseo de explorar una tercera vía (ni comunista ni capitalista) para el desarrollo, o las tensiones impuestas por los bloqueos económicos y políticos. Cardettini encuentra que la autodependencia es una "palabra engañosamente simple de definir" y muestra la limitación de las definiciones aceptadas tales como "confiar en sus propias fuerzas" o "contar con sus propios esfuerzos", particularmente cuando estos se toman como directivas de políticas. Por lo tanto, para integrar el concepto de autodependencia en una estrategia de desarrollo es necesario dar un contenido operacional a las directivas de políticas que estas implican con relación a áreas específicas, tales como el financiamiento, la producción alimenticia y la ciencia y la tecnología.

La autodependencia en ciencia y tecnología puede ser interpretada de tres formas diferentes:

a) Como la capacidad de tomar decisiones autónomas en materias de tecnología. Esto ha sido el enfoque sugerido por algunos autores latinoamericanos, que consideran esta autonomía de decisión como un prerrequisito para el desarrollo de una capacidad científica y tecnológica. ${ }^{2}$

En este caso no es necesario poseer la tecnologia para cubrir las necesidades tecnológicas dentro del país. La autonomía de decisión se refiere a la capacidad para definir los requerimientos tecnológicos, identificando alternativas disponibles en otras partes (desagregando sus componentes), y determinando la mejor forma de adquirir, incorporar y absorber la tecnología. Esto, a su vez, está relacionado con la capacidad para obtener y procesar la información sobre tecnología.

b) Como la capacidad para generar independientemente los elementos críticos del conocimiento técnico requerido para un producto o proceso particular. Los productos y los procesos están compuestos por muchos elementos de conocimiento técnico, algunos de los cuales pueden ser críticos porque son esenciales, o por causa de las dificultades para obtener acceso a ellos (p. ej. un catalizador en un proceso químico, un diseño en un equipo electrónico, etc.). Esta capacidad está estrechamente unida al desarrollo de las habilidades de diseño en ingeniería y no implica necesariamente que la totalidad del "elemento crítico" será producido dentro del país. Lo que se requiere

${ }^{2}$ Ver, por ejemplo, J. Sabato, Ciencia, Tecnologia, Desarrollo y Dependencia, Ed. Mensaje, Tucumán, Argentina, 1971. 
es la capacidad para diseñar el proceso o el producto (y en particular sus elementos críticos), para definir pautas y especificaciones para los componentes que serán manufacturados y para reunir los componentes en el diseño completo.

c) Como el potencial autónomo para producir dentro del pais los bienes y servicios considerados esenciales en la estrategia del desarrollo. Esto implica la posesión de las habilidades y el conocimiento técnico, así como también la capacidad para transformarlos en bienes y servicios. En este sentido, el pais sería capaz de "confiar en sus propias capacidades" si se le forzara a ello, si bien bajo condiciones normales no tratarfa de comprometerse en todas las actividades productivas que es capaz de realizar.

La primera interpretación del concepto de autodependencia puede ser extendida a una gran variedad de áreas. Es posible tener una capacidad autónoma de decisión con respecto a los medios para producir una mercancía determinada aun cuando las capacidades para producirla no están a su alcance. Esto requiere la posesión de un cuadro de profesionales y técnicos conocedores en cada área de interés, como también el acceso a la información que debe ser procesada para llegar a una decisión. Lo mismo puede aplicarse a la segunda interpretación, si bien el grado de conocimiento y habilidades requeridos serán muchos mayores, estarán directamente relacionados con las capacidades de diseño de ingeniería. La tercera interpretación del concepto de autodeterminación abarca las dos primeras y sólo puede ser conseguida en campos seleccionados que deben estar ligados a la estrategia del desarrollo. En este caso, no sólo se debe disponer de los cuadros y de la información, sino también de los medios actuales de producción (capacidad de manejo y de gerencia, facilidades de manufacturación, materias primas, etc.) que permitirán al país manejarse sin fuentes externas de aprovisionamiento.

Aun cuando referidas primariamente a la tecnología, estas interpretaciones de la autodependencia tienen lazos previos con la ciencia. Es necesaria la conducción de las actividades científicas para mantener una capacidad autónoma de decisión en la mayoría de los campos tecnológicos, particularmente en aquellos que se desarrollan a un rápido ritmo. Sin una base de científicos y profesionales activos es casi imposible seguir la cvolución de la tecnología y tener una percepción clara de las alternativas y opciones disponibles. Esto es más acentuado cuando la tecnologia se refiere al comportamiento de actividades específicas del país y para las cuales no se han desarrollado alternativas adecuadas.

Sin embargo, está claro que el concepto de autodependencia no se 
aplica a la conducción de la investigación científica como tal. La ciencia, considerada como un proceso para generar conocimiento, es una actividad internacional y su metodología, cánones y principios, como también sus descubrimientos, son universales. En este sentido ningún país puede ser autodependiente en la ciencia. Lo que es razonable es hablar acerca del desarrollo de las capacidades científicas que podrían proporcionar las bases para una autodependencia tecnológica.

El hecho de que estemos tratando con la autodependencia tecnológica no debe conducirnos a perder la perspectiva más amplia necesaria para colocarla en un contexto. Son necesarios fuertes compromisos políticos y transformaciones socioeconómicas internas para que un pais menos desarrollado pueda seguir una política de autodependencia en tecnología o en cualquier otra área.

Un prerrequisito para la autodependencia es la obtención de un grado significativo de autocontrol o de independencia nacional, entendiéndose por esto la libertad para establecer objetivos nacionales y para elegir los medios para cumplirlos. Esto implica un acto politico de afirmación y la posibilidad de mantenerlo - neutralizando las interferencias externas e internas-durante el tiempo necesario para consolidar las transformaciones y para construir los fundamentos de 1a estructura socioeconómica contemplada. Este acto de afirción debería incluir medidas para controlar los flujos de inversión, para modificar pautas de consumo, para dirigir la localización de las actividades sociales y productivas, y para dirigir el uso de los recursos naturales. ${ }^{3}$

Estas medidas deberían condicionar fuertemente la posibilidad de seguir una política de autodependencia en materias de ciencia y tecnología. Los modelos de inversión, consumo, localización y uso de los recursos determinan la naturaleza de la demanda por actividades científicas y tecnológicas. Por consiguiente, no es posible ser tecnológicamente autodependiente en tanto se mantengan políticas sensiblemente dilerentes para otras áreas de la estrategia del desarrollo. Todos estos factores deben ser combinados en un estilo coherente de desarrollo y una estrategia asociada con éste, que determinará hasta qué punto la política de autodependencia tecnológica tiene sentido. ${ }^{4}$

"Estos conceptos son desarrollados para el caso de Perú en J. Bravo Bresani, F. Sagasti, A. Salazar Bondi, El Relo de Perí en la Perspectiva del Tercer Mundo. Moncloa Editores, Lima, 1972.

"Para interpretaciones de la autodependencia en un contexto más amplio, ver The Cocoyoc Declaration, UNEP/UNCTAD, octubre 1974, y What to do, Informe de la Fundación Dag Hammarskjold, Uppsala, junio 1975. 
CAMBIOS EN EL ORDEN INTERNACIONAL Y SUS IMPLICACIONES PARA LA AUTODEPENDENCIA TECNOLÓGICA

La emergencia del subdesarrollo como un fenómeno histórico ha sido caracterizado por Furtado ${ }^{5}$ en los siguientes términos:

"Como una consecuencia de la rápida expansión de los nuevos mćtodos de producción de un pequeño número de centros que radian innovaciones tecnológicas, ha surgido un proceso tendientc a crear un sistema económico mundial. Es por eso que el subdesarrollo es considerado como una criatura del desarrollo, o más bien como la consecuencia del impacto de los procesos tecnológicos y de la división internacional del trabajo dirigida por $\mathrm{cl}$ pequeño número de sociedades que se unieron a la Revolución Industrial en el siglo diez y nueve. Las relaciones resultantes entre estas sociedades y las áreas subdesarrolladas envuelven formas de dependencia que difícilmente pueden ser superadas. La dependencia estaba basada en un comienzo en una división internacional del trabajo, en la cual los centros dominantes reservaban para ellos mismos las actividades económicas que concentraban el progreso técnico. En la fase siguiente, la dependencia era mantenida controlando la asimilación del nuevo proceso tecnológico a través de la instalación de actividades productivas dentro de las economías dependientes, todo bajo el control de grupos integrados dentro de las economías dominantes" (p. $\mathrm{XVI})$.

Existe evidencia cle que la naturaleza del proceso descrito por Furtado en lo que respecta a las relaciones entre las economias dominantes y dominadas continúa y está cambiando a un ritmo rápido. La fase siguiente en este proceso consiste en un vuelco hacia el control de los recursos financieros ${ }^{6}$, y ahora estamos siendo testigos de la transición hacia el uso del conocimiento tecnológico como el principal vehículo para mantener las relaciones de dominación. De este modo ha habido un desplazamiento de los medios de control de los países desarrollados hacia los subdesarrollados desde las materias

${ }^{5} \mathrm{C}$. Furtado, Obstacles to development in Latin America, Anchor Books, New York, 1970.

-Ver M. C. Tavares, Da substituição de Importaçōes ao capitalismo financiero, Zahar Editores, Rio de Janeiro, 1972. 
primas a los sectores productivos, el capital y servicios financieros, $y$ ahora hacia la tecnología. En este proceso de mutación de las relaciones dominantes, la tecnología ha estado siempre detrás como un factor condicionante, pero ahora ha emergido finalmente a la luz, en parte a causa de las dinámicas internas de la evolución del sistema económico capitalista, y en parte a causa del control creciente de los países menos desarrollados sobre los medios a través de los cuales los países desarrollados ejercieron su dominación en el pasado.

Dos extractos de declaraciones de líderes de países industrializados del occidente pueden mostrar hasta qué punto está llevándose a cabo esta transformación de las relaciones dominantes. En una intervención del Secretario de Estado de los Estados Unidos, Dr. Kissinger, a la Asamblea General de la Organización de Estados Americanos (el 20 de abril de 1974), se dijo que:

"La transferencia de la ciencia y la tecnología, puede ser un cuello de botella más importante en el esfuerzo de desarrollo que el capital. Los Estados Unidos, como una nación tecnológicamente avanzada, reconoce una responsabilidad especial en este aspecto. Creemos que las inversiones privadas normalmente son el vehículo más eficiente para la transferencia en gran escala de estos recursos, pero los gobiernos pueden facilitar la transferencia de la tecnología avanzada para estimular un desarrollo balanceado" (énfasis añadido).

En un discurso efectuado en la sesión del parlamento europeo a principios de 1975, el señor Ortoli, presidente de la Comisión de las Comunidades Europeas, expresó que:

"A la vez que continuar con la ayuda financiera que es indispensable para ciertos países, debemos, cada vez que sea posible, trabajar para la cooperación basada en lazos económicos a largo plazo, que son un instrumento mejor para el progreso y la solidaridad que cualquier tratado. Mientras respetamos los propios objetivos de nuestros socios, debemos combinar nuestra tecnologia y conocimiento, nuestros mercados, en ciertos casos nuestro capital y nuestros productos, en particular los agrícolas, con los recursos de nuestros socios y su deseo de aprovechar esta nueva situación para impulsar su desarrollo" (énfasis añadido).

El énfasis puesto en la tecnología en ambos casos es bastante sorprendente y la reflexión de la prominencia que va a adquirir en el futuro, particularmente a medida que los países del Tercer Mundo aumentan su control sobre sus propios recursos naturales. Habrá una tendencia a usar el acceso a la tecnología como la principal palanca 
en las relaciones dominantes Norte-Sur, con el uso subsidiario de los alimentos, y en algunos casos del capital, para complementarlo. ${ }^{7}$

Bajo estas circunstancias, la importancia de la autodependencia tecnológica no puede ser subestimada. Existe una urgente necesidad de tomar medidas que puedan proporcionar a los países del Tercer Mundo un mínimo de medios para confrontar esta nueva situación. De hecho la posibilidad de seguir un camino independiente hacia el desarrollo será determinada por el grado hasta el cual el país es tecnologicamente autodependiente. Esto requiere el establecimiento de una estrategia total para la autodependencia tecnológica, definiendo las áreas en las cuales serán aplicadas cada una de las diferentes interpretaciones del concepto, sus interrrelaciones y el tiempo en el cual pueden ser completadas.

Hemos visto antes que la autodependencia tecnológica no puede ser alcanzada fuera del contexto de un estilo y una estrategia de desarrollo autónomo. Por lo tanto, los temas de la autodependencia tecnológica y los estilos alternativos de desarrollo interactúan entre ellos, hasta el punto de que no pueden ser considerados independientemente.

La autodependencia tecnológica es incompatible con un estilo de desarrollo que mantenga el modo actual de inserción dependiente de los países subdesarrollados en el sistema económico mundial. Ella puede ser realizada totalmente sólo dentro del contexto de un estilo y estrategia de desarrollo que modifique significativamente la posición internacional del país. Sin embargo, efectuar los cambios que permitirían a los países menos desarrollados seguir su propia vía hacia el desarrollo requiere una acción concertada, puesto que muchos de ellos no tienen el poder de llevar a cabo por sí mismos los cambios necesarios en la situación internacional: se requieren esfuerzos colectivos para seguir estrategias independientes de desarrollo.

Existe una aparente contradicción en la necesidad de colaborar con otros en la búsqueda de la autodependencia, si bien esta contradicción desaparece cuando la colaboración es considerada como un proceso de concentración de fuerzas entre los países que tengan los mismos intereses básicos, esto es, los países subdesarrollados. En principio, cualquier forma de alianza implica ciertas limitaciones en la libertad individual, pero estas limitaciones no necesitan interferir con la principal orientación elegida para el proceso de desarrollo del pais.

Resumiendo, el logro de la autodependencia en materia de tecnología requiere que se siga una estrategia de desarrollo independiente.

${ }^{7}$ Sobre estos temas ver M. Halty, Towards a new Technological Order?, presentado en el Scminario de la OECD sobre Ciencia, Tecnología y Subdesarrollo cn un mundo cambiante, París, abril 1975. 
También, la autodependencia tecnológica condiciona la posibilidad de seguir una estrategia independiente. Ambos implican la necesidad de romper la forma dependiente de inserción de los paises en desarrollo dentro de la economía mundial y la búsqueda de nuevos caminos para vincularse con ella. Finalmente, los cambios significativos en las formas de inserción de los países subdesarrollados sólo pueden ser alcanzados a través de una acción concertada por parte de aquellos que obtienen beneficios de estos cambios. Esto proporciona un argumento poderoso para promover la cooperación entre los países subdesarrollados, particularmente cuando está relacionada con la búsqueda de la autodependencia tecnológica.

\section{LA DISTRIBUCIÓN DEL ESFUERZO CIENTÍFICO Y TECNOLÓGICO $X$ SU IMPACTO EN LA AUTODEPENDENCIA}

En la segunda mitad del siglo veinte es posible observar un proceso de concentración de las fuentes del cambio tecnológico, que es impuesto a nivel mundial y a un ritmo siempre creciente -por un número relativamente pequeño de países avanzados y grandes empresas. Las características de este proceso son: a) El alto grado de interdependencia entre los intereses militares y los intereses industriales, a los cuales se añadió la industria del espacio en los años sesenta, que influyen en la naturaleza del progreso tecnológico; y b) La creciente interconexión entre los intereses relacionados con la investigación y el desarrollo de las grandes corporaciones multinacionales, una de cuyas características centrales es lo que se ha dado en llamar el "sistema industrial global". Ambas características muestran que la velocidad y dirección del cambio técnico en la actualidad están determinadas hasta un muy alto grado por intereses que no tienen nada que ver con las aspiraciones y las metas de los países subdesarrollados. Aún más, el grado de concentración se está tornando tan elevado que un limitado número de ejecutivos de grandes corporaciones y empleados gubernamentales de países desarrollados pueden ejercer una influencia decisiva en la naturaleza del cambio técnico a nivel mundial.

Al mismo tiempo que este proceso de concentración está en marcha, la masa crítica mínima que supone cualquier esfuerzo viable en el campo de la ciencia y la tecnología está aumentando. Sobre la base de un número mínimo de institutos de investigación de diversos tipos, Herrera ${ }^{8}$ llega a la conclusión de que en 1970 un mínimo de

${ }^{8}$ A. Herrera, Ciencia y Política en América Latina, Siglo xxi, México, 1971. 
cien millones de dólares se requeriría para mantener un sistema científico y tecnológico viable. Él no incluye los costos de la transformación de los resultados de la investigación en productos o procesos, y desde que sus cálculos fueron hechos, el costo de las actividades científicas y tecnológicas ha aumentado sin duda. Otras estimaciones colocan el umbral de los gastos para un sistema científico y tecnológico viable en el uno por ciento del producto nacional bruto. Estas cifras dan solamente una idea general sobre los requerimientos mínimos, e indican que pocos países subdesarrollados tienen ahora la capacidad para comprometerse en la construcción de un sistema científico y tecnológico independientemente viable. Cuando se examinan cifras de mano de obra calificada, éstas conducen a una conclusión similar.

Además, a causa del pequeño tamaño de los mercados internos de la mayoría de los paises subdesarrollados, existen también limitaciones al poder de negociación que pueden ejercer cuando entran en tratos con los proveedores de tecnología de los países desarrollados. Aún más, el alto costo y las dificultades para obtener acceso a las fuentes de información, que mejorarían su capacidad de negociación, hacen prácticamente imposible para la mayoría de los países subdesarrollados el adquirir por sí mismos la información relevante.

La necesidad de alterar la distribución mundial de los recursos para la ciencia y la tecnología, y de romper el alto grado de concentración de las fuentes de cambio tecnológico, la necesidad de aumentar la masa crítica mínima para poder tener sistemas científicos y tecnológicos viables, y la necesidad de mejorar la posición negociadora para la adquisición de tecnología, conduce al imperativo de la cooperación en el campo de la ciencia y tecnología entre los paises subdesarrollados. Será imposible seguir una politica de autodependencia tecnológica si no se cumple esta condición, porque los obstáculos que se presentarían serían demasiado grancles para ser superados individualmente.

Sin embargo, la experiencia muestra que los acuerdos de cooperación son relativamente más fáciles de alcanzar cuando hay envueltos asuntos de naturaleza puramente científica, pero que cuando los programas de cooperación encierran actividades científicas y tecnológicas que pueden tener applicación económica directa, es más difícil obtener un acuerdo. De este modo, los programas conjuntos efectivos requerirán un nuevo espíritu de coopcración entre los países del Tercer Mundo, sobre la base de cuáles ganancias de corto plazo para un pais en particular deberán ser consideradas como desequilibrios temporales a lo largo del camino de los esfuerzos colectivos hacia la autodependencia tecnológica.

Sólo después que un cierto grado de cohesión entre los países 
subdesarrollados ha sido alcanzado a través de arreglos concretos de colaboración será posible comprometerse en el proceso de reestructurar la inserción de un país determinado dentro del sistema científico y tecnológico mundial. En efecto, esto implica una estrategia en dos etapas, en la cual la creciente cooperación del Tercer Mundo se ve como un prerrequisito para nuevas formas de colaboración entre paises subdesarrollados y desarrollados.

EL POSIBLF. CONTENIDO DE LOS ACUERDOS DE COOPERACIÓN

Una vez que ha sido aceptado el imperativo de la cooperación del Tercer Mundo para la consecución de la autodependencia tecnológica, la tarea consiste en identificar áreas adecuadas de colaboración, para obtener compromisos políticos, y para diseñar programas es. pecíficos. Entre las áreas posibles donde tales programas pueden ser instituidos, tenemos:

- Actividades que requieren una masa crítica mínima para ser realizadas. Esto incluye investigación y desarrollo, para lo cual es necesario contar con profesionales, equipos y financiamiento a un nivel bajo en el cual estas actividades no son viables. En estos campos es imposible intervenir individualmente y los esfuerzos de cooperación son indispensables.

- Actividades científicas y tecnológicas en las cuales hay economías de escala (sistema de información, programas de entrenamiento, capacidad de ingeniería, investigación y desarrollo en común). En este caso la cooperación internacional no es absolutamente necesaria, pero implica muchos beneficios que la hacen altamente deseable.

- Actividades que deben poseer una dimensión internacional para tener sentido. Esto incluye acciones comparativas y conjuntas que no tienen sentido cuando se consideran en un solo país. Un ejemplo seria el establecimiento de sistemas comparativos de información sobre los términos y condiciones para la transferencia tecnológica, que aumentaría el poder de negociación de los países compradores de tecnología. Esto puede extenderse a acuerdos sobre estrategias comunes para negociaciones con proveedores de tecnología y a la adopción de posiciones conjuntas ante las corporaciones multinacionales, instituciones financieras multilaterales y otras organizaciones semejantes. 
- Problemas comunes a más de un país, relacionados con zonas geográficas que se extienden más allá de las fronteras nacionales. Esto incluye la investigación de las condiciones ecológicas, la explotación de los recursos naturales, el uso de los sistemas hídricos, y así sucesivamente. En este caso, la existencia de un problema común proporciona al país la posibilidad de unir sus fuerzas con otros en la realización de actividades científicas y tecnológicas.

- Grandes empresas, en las cuales es necesario compartir riesgos entre diversos paises a causa de la magnitud de los recursos requeridos. Esto ha sido el caso de inversiones en energía nuclear, computadores, telecomunicaciones por satélite, etc., en las cuales pocos paises individuales -aun si son capaces de financiar el programa por sí mismos- desean asumir el riesgo solos.

Si la cooperación tecnológica se realiza dentro del contexto de una integración política y económica más amplia, emergen campos adicionales para la colaboración internacional entre los países menos desarrollados ${ }^{9}$. Entre éstos, tenemos la explotación común de ciertas tecnologías que, a causa de su graduación, son viables sólo en términos de un mercado más expandido. La armonización de las políticas nacionales económicas para la realización de la autodependencia puede ser incluida también aquí, así como la búsqueda de tecnologías para proyectos conjuntos de desarrollo económico. Los beneficios plenos de la creciente cooperación para la autodependencia tecnológica pueden ser sólo realizados cuando se consideran como parte integral de un proceso más amplio de cooperación económica y política.

El establecimiento de un sistema de cooperación en materias de ciencia y tecnología puede requerir una reformulación de conceptos, tales como "región", que ha sido usado tradicionalmente para definir grupos entre los países subdesarrollados. Las "regiones" centradas en problemas pueden ser definidas en términos de la necesidad de embarcarse en programas conjuntos para la solución de problemas especificos tecnológicos. Por ello, una "región" para la cooperación científica y tecnológica en el Tercer Mundo puede abarcar países que están geográficamente dispersos, que no comparten la misma herencia cultural y que poseen diferentes sistemas políticos. Las características comunes que los agrupan serían las áreas de problemas y la voluntad de unirse para llevar a cabo actividades que los ayudarían a solucionarlos.

'Ver F. Sagasti, "Investigación económica y politica tecnológica, el caso del Pacto Andino", cn Comercio Exterior (México), enero 1975, y en Revista de la Integración, Buenos Aires, No 18, enero-marzo 1975. 
plio. Por lo tanto, debo dirigir mis observaciones a los programas multilaterales.

La estructura existente de las organizaciones multilaterales internacionales, muchas de las cuales reúnen tanto países desarrollados como subdesarrollados, cumple funciones útiles en una variedad de campos.

Sin embargo, existe la necesidad de complementar sus actividades con nuevas formas de organización que tendrían mayor flexibilidad, operarían a un costo menor, y responderían más directa y rápidamente a las necesidades de cooperación entre los países subdesarrollados para la obtención de la autodependencia tecnológica. Una posibilidad es estructurar un sistema dual consistente en un acuerdo general para una cooperación multilateral más amplia, junto con una variedad de acuerdos multilaterales específicos.

La idea sería establecer una asociación internacional con una amplia participación de los países subdesarrollados de todas las regiones del mundo ${ }^{11}$. Su carácter de miembro implicaría compartir los costos de mantenimiento de un pequeño equipo central, cuya principal tarea sería identificar, estructurar y lanzar proyectos cientificos y tecnológicos para ser llevados a cabo por países miembros. Los proyectos podrían comprender investigación, adaptación tecnológica, negociaciones con los proveedores de tecnología, entrenamiento y otras actividades relacionadas con la búsqueda de la autodependencia tecnológica. No todos los países participarían en cada proyecto, si bien se esperaría que cada país participaría por lo menos en un proyecto durante un razonable período de tiempo. La asociación podría establecerse a través de un amplio acuerdo multilateral firmado por todos los países miembros, y los proyectos serían propiciados a través de acuerdos multilaterales específicos entre los paises interesados en ellos.

El personal central consultaría con las instituciones relevantes en los países miembros para poder determinar las prioridades para la identificación y el diseño de los proyectos de investigación. Se contempla el que un pequeño grupo de profesionales altamente calificados, designados por un plazo fijo (p. cj. cinco años), conformarían el equipo central. Estarían asesorados por consultores que trabajarian por períodos breves. El equipo central por sí mismo no estaría dedicado directamente a la investigación con su actividad principal, si bien podría participar activamente en algunos proyectos específicos. El financiamiento para el equipo central estaría ase-

"E. grupo de paises no alineados constituiria una base natural para Ia organización de esta asociación y en la reunión de agosto de 1975 de los Ministros de Relaciones Exteriores en Lima, ellos aprobaron el establecimiento dc un esquema similar al sugerido aquí. 
gurado por las contribuciones de los países miembros, y posiblemente por fondos de organismos internacionales y agencias donantes.

Por lo tanto, no sería una merma significativa en los recursos externos de los países miembros. Está claro que el equipo central estaría localizado en un país del Tercer Mundo. Un consejo supervisor elegido por los países miembros supervigilaría las funciones del equipo central.

Los proyectos específicos se realizarian en instituciones de los países participantes en forma descentralizada. Los proyectos serían temporales y existiría un comité coordinador -que dirigiría la orientación de cada proyecto- conformado por un representante de cada país participante. Si fuera necesario, habría también un coordinador ejecutivo del proyecto, responsable frente al comité. De esta ma. nera no se crearía alrededor de los proyectos una estructura organizativa permanente. En un momento dado habrán varios proyectos específicos bajo ejecución, otros en el período de gestación, y aun otros que deberían ser completados. Los arreglos organizativos dependerán de la naturaleza y enfoque de los problemas que se abarcarían, puesto que algunos tal vez requirirían la existencia de un laboratorio central y otros podrían ser manejados en forma totalmente descentralizada. Debe mantenerse gran flexibilidad a este respecto.

El sistema planteado para la cooperación del Tercer Mundo para la búsqueda de la autodependencia tecnológica conduciría a un proceso de identificación de intereses comunes, organizando activida des específicas de cooperación, y aplicando los resultados de acuerdo con los intereses y los objetivos de un pais dado. Esto generaría un proceso de forja, división y reestructuración de eslabones de acuerdo a las cambiantes necesidades y capacidades con el propósito de lograr la autodependencia en el campo de la tecnología. A menos que se tomen acciones concretas en el corto plazo por parte de los países subdesarrollados -organizando un sistema de cooperación como el que se ha propuesto aquí, o poniendo en efecto cualquier otra forma de arreglos de colaboración- la autodependencia tecnológica podría adquirir el mismo significado que la autodependencia sexual. 